\title{
Von Willebrand Factor-Cleaving Protease Activity in Thrombotic Microangiopathy: First Report From Iran
}

\author{
Mohammadreza Ardalan ${ }^{1, *} ;$ Parisa Rezaeifar ${ }^{1}$ \\ ${ }^{1}$ Chronic Kidney Disease Research Center, Tabriz University of Medical Sciences, Tabriz, IR Iran \\ ${ }^{*}$ Corresponding author: Mohammadreza Ardalan, Chronic Kidney Disease Research Center, Tabriz University of Medical Sciences, Tabriz, IR Iran. Tel: +98-9141168518, Fax: +98- \\ 4113366579, E-mail: ardalan34@yahoo.com
}

Received: March 12, 2014; Revised: May 10, 2014; Accepted: May 13, 2014

\begin{abstract}
Background: Thrombotic microangiopathy (TMA) is a rare but devastating small vessels disorder that is characterized by intravascular platelet thrombi, thrombocytopenia, and various degrees of organ ischemia and anemia, which is due to erythrocyte fragmentation in microcirculation.

Objectives: The Aim of this study was to determine the von Willebrand factor-cleaving protease (ADAMTS13) activity during the acute phase of TMA. We also investigated inhibiting antibodies against ADAMTS13 in these patients.

Patients and Methods: In a collaborative work with Mario-Negro institute of pharmacological research in Bergamo-Italy, we registered the clinical and laboratory data, collected the serum samples, and transferred the samples to the laboratories. Serum samples were taken before the start of plasmapheresis or at least 15 days after the final exchange.

Results: We recruited 40 patients ( 14 males and 26 females) with the mean age of $46.12 \pm 17.26$ years. The mean activity of ADAMTS13 was $34.58 \% \pm 21.83 \%$. Two patients had inhibitory antibodies against ADAMTS13 with profound deficiency of ADAMTS13 activity $(<6 \%)$. Infectious diseases were the most common underlying condition, followed by systemic lupus erythematous.

Conclusions: Majority of patients had an underlying condition and had various ADAMTS13 activity. The presence of inhibiting antibodies and accompanied complete deficiency of ADAMTS13 activity is an indicator of severity.
\end{abstract}

Keywords:Purpura; Syndrome; Thrombotic Microangiopathy

\section{Background}

Thrombotic microangiopathic (TMA) is a rare but devastating disorder of small vessels that is characterized by intravascular platelet thrombi, thrombocytopenia, and various degrees of organ ischemia and anemia, which is due to erythrocyte fragmentation in microcirculation (1). In thrombotic thrombocytopenic purpura (TTP), systemic microvascular aggregation of platelets mainly causes ischemia in the brain. In the hemolytic-uremic syndrome (HUS), platelet-fibrin thrombi predominantly occlude the renal circulation (2). Adults with major central neurological involvement are labeled mainly as TTP (1). TTP and HUS are not distinct syndromes and their essential diagnostic criteria are the same. Although neurologic abnormalities such as confusion, focal deficits, seizures, or coma are commonly considered characteristics of TTP and renal failure is more common in HUS, some patients with TMA have both neurologic and renal involvement. Prompt recognition of TTP is important because the disease responds well to plasma-exchange while high mortality ensues when it remain untreated. Nevertheless, recognition of TTP can be difficult because of its different features and lack of specific diag- nostic criteria. Consistent abnormalities are red cell fragmentation and thrombocytopenia (3). Endothelial damage due to toxins and inhibitory antibodies to von Willebrand factor (vWF)-cleaving protease (ADAMTS13), which impair endothelial defense against complement activation, has a central role in pathogenesis (4). Discovery of ADAMTS13 has offered a new insight into the pathogenesis of TMA (4). ADAMTS13 is a metalloprotease that cleaves VWF at the Tyr1605-Met1606 bond in the central A2 domain. This cleavage progressively converts the vWF polymer to smaller multimers that are less adhesive. When ADAMTS13 activity is deficient, vWF polymers are not cleaved, which results in accumulation of hyperactive intact forms of vWF that causes platelet aggregation and microvascular thrombosis (5). During the course of plasma exchange therapy, increasing ADAMTS13 activity level is associated with clinical and hematologic improvement (6). Severe deficiency of ADAMTS13 $(<6 \%)$ is observed in genetic mutations or in the presence of inhibiting autoantibodies. Occasionally, low levels of ADAMTS13 might be observed in disseminated intravascular coagulopathy, liver disease, or sepsis (7). 


\section{Objectives}

We aimed to measured ADAMTS13 activity and status of their ADAMTS13-inhibiting antibody during acute phase of TMA.

\section{Patients and Methods}

All patients with the diagnosis of TMA were registered by Chronic Kidney Disease Research Center of Tabriz University of Medical Sciences since 2003 to 2011. The diagnosis of acute TMA was made based on at least three of the following criteria: (a) thrombocytopenia with no other apparent cause; (b) Coombs-negative hemolytic anemia with schistocytes; (c) high serum levels of lactate dehydrogenase (LDH); and (d) signs or symptoms of target organ involvement including's kidney or central nervous system involvement (8). Demographic, clinical, and laboratory parameters of all studied patients were entered in the standard forms.

Serum samples were obtained from all individuals during the acute phase before the first plasma exchange or at least two weeks after the last therapeutic plasma infusion or plasma exchange, and were stored at $-80^{\circ} \mathrm{C}$ in tubes containing trisodium citrate as anticoagulant. Patients received detailed information on purposes of the study and signed a written informed consent. According to an agreement and approval by Mario Negri Institute of Pharmacological Research in Bergamo, Italy, all collected samples were sent to that institute while they were kept at $<0^{\circ} \mathrm{C}$ during the transfer and their quality were checked by the institute at the time of reception. ADAMTS13 activity was measured as described by Gerritsen et al. $(9,10)$. The samples with activity levels of $<$ $30 \%$ and $<6 \%$ were classified as deficient and complete deficient ADAMTS13 activity, respectively. The activity levels of $>30 \%$ were considered normal or near normal results. Inhibiting antibodies to ADAMTS13 activity were searched in all patients by measuring residual activity in a 50:50 mixture of heat-inactivated patient's plasma and normal pooled human plasma. Heat-inactivated plasma was obtained after incubation at $56^{\circ} \mathrm{C}$ for 60 minutes to completely neutralize endogenous ADAMTS13 activity. A patient would be considered to have ADAMTS13 inhibitor (1 Bethesda unit) if the residual activity of the protease in the plasma mixture were less than $50 \%$ of the control mixture (a 50:50 mixture of heat-inactivated and unheated normal plasma) $(9,11)$.

We used a simple descriptive analysis of our data.

\section{Results}

From total number of 40 studied patients, 14 (35\%) were male and 26 (65\%) were female. The mean age of patients was $46.12 \pm 17.26$ years (range, 18-87). The demographic characteristics of the patients are summarized in Table 1.

In terms of underlying disease, infectious diseases were the most prevalent presumptive cause of TMA (50\%). Among the infections, urinary tract infection (UTI) was the most common underlying cause. The second cause was gastroenteritis and diarrhea (16.7\%). Infections sinusitis, bacterial pneumonia, and in one case, hydatid cyst were the concomitant infections. Pregnancy was the second presumptive causes of TMA. Systemic lupus erythematous (SLE) was the most common autoimmune diseases (85\%) and the mean activity of ADAMTS13 activity in this group was $26 \%$. TMA was also detected in a patient with Goodpasture disease. Another patient developed TMA immediately after myocardial infarction while he was taking Clopidogrel (Plavix) for a year; ADAMTS13 activity level in this patient was $25 \%$. We also detected TMA in a 38-year-old woman who was HBsAg positive and had started taking oral contraceptive recently. Acute myeloid leukemia, multiple myeloma, and esophageal squamous cell carcinoma were the concomitant malignancies in three patients. Malignant

Table 1. Clinical and Laboratory Data of Patients With Thrombotic Microangiopathic Anemia a,b

\begin{tabular}{|c|c|}
\hline Variable & Values \\
\hline \multicolumn{2}{|l|}{ Prod Rome Situation } \\
\hline Neurologic Manifestations & $17(42.5)$ \\
\hline Diarrhea & $1(2.5)$ \\
\hline Both & $3(7.5)$ \\
\hline None & $19(47.5)$ \\
\hline \multicolumn{2}{|l|}{ Associated Disease } \\
\hline Infection & $12(30)$ \\
\hline Pregnancy/Per partum Period & $8(20)$ \\
\hline Autoimmune Disease & $7(17.5)$ \\
\hline $\begin{array}{l}\text { Hematologic and No hematologic Malig- } \\
\text { nancies }\end{array}$ & $3(7.5)$ \\
\hline Drug & $2(5)$ \\
\hline Unknown & $5(12.5)$ \\
\hline Others & $3(7.5)$ \\
\hline Hemoglobin Concentration, $\mathrm{g} / \mathrm{L}$ & $90.4 \pm 19.1$ \\
\hline Platelet Count $/ \mu \mathrm{L}$ & 42600 \\
\hline LDH, U/L $\mu$ kat $/ \mathbf{L}$ & 32.50 \\
\hline Creatinine, $\mu \mathrm{mol} / \mathbf{L}$ & 379.24 \\
\hline \multicolumn{2}{|l|}{ Complement, g/L } \\
\hline C3 (Nl: 83-177) & $0.12 \pm 0.05$ \\
\hline C4 (Nl: 15-45) & $0.03 \pm 0.017$ \\
\hline \multicolumn{2}{|l|}{ ADAMTS13 activity } \\
\hline$\leq 30 \%$ & $17(42.5)$ \\
\hline$>30 \%$ & $23(57.5)$ \\
\hline ADAMTS13 inhibitor & $\begin{array}{l}\text { Positive results in } \\
2 \text { patients; suspi- } \\
\text { cious in } 1 \text { patient }\end{array}$ \\
\hline
\end{tabular}


hypertension was the precipitating cause in two patients with ADAMTS13 activity of $23 \%$ and $50 \%$. We also detected TMA in a patient who was hospitalized because of subarachnoid hemorrhage and intracerebral hemorrhage without any episode of malignant hypertension. In general, the mean activity level of ADAMTS13 was $34.58 \% \pm$ $21.83 \%$. Two patients (5\%) had complete deficiency of ADAMTS13 activity because of inhibiting antibodies. In one of them, pregnancy was the probable precipitating factor and she died because of severity of her disease. Another patient had no apparent condition.

\section{Discussion}

In our study, majority of patients had an underlying condition during the clinical diagnosis of TMA. Infectious disease, pregnancy, and autoimmune disease were the most common precipitating factors. ADAMTS13 had variable activity levels. We found that even in those patients who had a relevant condition such as malignant hypertension, the low level of ADAMTS13 could be the precipitating cause for TMA. The presence of inhibiting antibodies is an indicator of severity. It is often difficult to differentiate between the two clinical syndromes of TTP and HUS. Early in 1998, Furlan et al. reported 23 patients who were diagnosed with HUS that had some subnormal ADAMTS13 activity (12). Choi et al. in 2011 studied ADAMTS13 activity and ADAMTS13 gene mutation in children with hemolytic uremic syndrome. They found five ADAMTS13 gene mutations without deficiency of ADAMTS13 activity in three patients with HUS and they could not answer whether these mutations without reduced ADAMTS13 activity were a predisposing factors (13). Bianchi et al. determined ADAMTS13 activity level in various conditions that were accompanied by thrombocytopenia such as severe sepsis or septic shock, heparin-induced thrombocytopenia, and idiopathic thrombocytopenic purpura. They noticed that mild and even moderate decrease in ADAMTS13 activity was common in these conditions (7). Severe deficiency of ADAMTS13 activity (<5\%-10\%) is a specific finding in clinically diagnosed TTP $(2,14)$. Tsai and Lian (15) observed severe ADAMTS13 deficiency in all of 37 patients with acute TTP and Furlan et al. observed this situation in 26 out of 30 patients (12). Another study reported a severe deficiency of ADAMTS13 in 47 of 66 patients (71\%) with idiopathic or secondary TTP (7).

In 1997, a complete deficiency of ADAMTS13 activity was reported by Furlan et al. in four patients with chronic relapsing TTP (16). In a literature review on ADAMTS13, Mannucci and Peyvandi stated that it is unnecessary to assay ADAMTS13 for the diagnosis of acute TTP and activity measurement is an index to predict the relapse (17). The Oklahoma TTP-HUS registry believes that relapses occur mainly in patients with severe autoantibody-mediated ADAMTS13 deficiency and it most often occurs during the first year of the TTP attack $(10,18)$. Furlan et al. and Tsai et al. reported complete deficiency of ADMATS13 in patients who had a circulating IgG inhibitor against the ADAMTS13 $(12,15)$, which was compatible with the results of the present study regarding two patients with severe deficiency of ADAMTS13 activity level and presence of inhibiting autoantibodies. Infections are reported as underlying conditions that induce TTP (19);; some of these infections are pulmonary tuberculosis, cytomegalovirus pneumonitis, mucocutaneous herpes simplex infection, ventilatorassociated pneumonia, UTI, gram-negative sepsis, central line infections with staphylococcal bacteremia, and cellulites $(4,20,21)$. Ardalan et al. reported cases of TMA in renal transplantation as a result of Parvovirus B19 and aspergillosis $(21,22)$ Crimean-Congo hemorrhagic fever has been reported as a cause of TMA and acute renal failure (23). In our study, UTI (with and without renal stones), gastroenteritis and diarrhea, bacterial sinusitis, pneumonia, and hydatid cyst were presumptive underlying causes. TTP has been reported in association with SLE, might precede or follow SLE, has similar clinical symptoms, and the levels of ADAMTS13 activity are decreased in both conditions (24). Yang et al. reported a patient with typical TTP who finally ended with the diagnosis of SLE with a good response to the initial plasmapheresis (25). In our cohort, we had six patients with SLE as the underlying cause of TMA and all of them improved with plasmapheresis. TTP might be detected during pregnancy or peripartum period. Characteristic signs of TTP might occur in patients with severe preeclampsia, which is named HELLP syndrome (hemolysis, elevated liver-enzyme levels, and a low platelet count) and make it difficult to distinguish from TTP (26). Molvarec et al. found that although plasma level of vWF were significantly higher in patients with preeclampsia in comparison with healthy pregnant and non-pregnant women, ADAMTS13 activity was normal in majority of these patients (27). We reached the same findings in our study. TTP affects one in 1600 to one in 5000 patients who receive ticlopidine; however, little is known about the pathogenesis of this complication. In a study by Tsai et al. seven patients with ticlopidine-associated TTP had severe decrease in ADAMTS13 levels (5). In our study, we had one case of ticlopidine-associated TMA in whom ADAMTS13 activity was slightly decreased. van den Born et al. reported that ADAMTS13 was deficient in malignant hypertensioninduced TMA, probably because of the release of vWF from stimulated endothelium (28). Finally, although kidney is the main target organ, other organs might be affected too. Cardiac complications occur in 3\% to $10 \%$ of patients with complement-mediated HUS and as a consequence of microangiopathic injury in the coronary microvasculature, sudden cardiac death could happens (29).

\subsection{The Limitation of Our Study}

We did not measure the ADAMTS13 activity during clinical follow-up and we did not study the activity of complement inhibitory factors that might underlie the clinical picture of TMA (either TTP or HUS). 
We concluded that TMA is an important clinical diagnosis in our daily clinical practice, particularly in tertiary medical centers, and its timely and correct treatment could be lifesaving. Inhibitory antibodies against the ADAMTS13 could lead to sever and fatal disease in other condition. Underlying conditions should be sought thoroughly; infection, particularly UTI, pregnancy, and autoimmune disease are important underlying conditions.

\section{Acknowledgements}

We express our sincere thanks to Professor Giuseppe who kindly offered us the possibility of sending the samples to their esteemed center according to an agreement and approval by Mario Negri Institute of Pharmacological Research in Bergamo, Italy. Since 2003, we sent or personally carried the samples to their laboratories. Dr. Marina Noris and Dr. Elena Bresin greatly helped us to perform measurement.

\section{Funding/Support}

We did not receive any financial support from any institution or organization in Iran. Mario-Negri Institute of Pharmacological Research in Bergamo, Italy, performed all the measurements for our patient free and kindly sent the result of all examined patients.

\section{References}

1. Ardalan MR. Review of thrombotic microangiopathy (TMA), and post-renal transplant TMA. Saudi J Kidney Dis Transpl. 2006;17(2):235-44.

2. Moake JL. Thrombotic microangiopathies. $N$ Engl J Med. 2002;347(8):589-600.

3. George JN. Clinical practice. Thrombotic thrombocytopenic purpura. NEngl J Med. 2006;354(18):1927-35.

4. Ardalan M. Urinary tract infection associated with thrombotic microangiopathy. Nephrourol Mon. 2014;6(1).

5. Tsai HM, Rice L, Sarode R, Chow TW, Moake JL. Antibody inhibitors to von Willebrand factor metalloproteinase and increased binding of von Willebrand factor to platelets in ticlopidine-associated thrombotic thrombocytopenic purpura. Ann Intern Med. 2000;132(10):794-9.

6. Tsai HM. Thrombotic thrombocytopenic purpura: a thrombotic disorder caused by ADAMTS13 deficiency. Hematol Oncol Clin North Am. 2007;21(4):609-32.

7. Bianchi V, Robles R, Alberio L, Furlan M, Lammle B. Von Willebrand factor-cleaving protease (ADAMTS13) in thrombocytopenic disorders: a severely deficient activity is specific for thrombotic thrombocytopenic purpura. Blood. 2002;100(2):710-3.

8. Allford SL, Hunt BJ, Rose P, Machin SJ, Thrombosis Task Force BCFSIH, Haemostasis.. Guidelines on the diagnosis and management of the thrombotic microangiopathic haemolytic anaemias. BrJ Haematol. 2003;120(4):556-73.

9. Gerritsen HE, Turecek PL, Schwarz HP, Lammle B, Furlan M. Assay of von Willebrand factor (vWF)-cleaving protease based on decreased collagen binding affinity of degraded vWF: a tool for the diagnosis of thrombotic thrombocytopenic purpura (TTP) Thromb Haemost. 1999;82(5):1386-9.

10. George JN, Vesely SK, Terrell DR. The Oklahoma Thrombotic
Thrombocytopenic Purpura-Hemolytic Uremic Syndrome (TTPHUS) Registry: a community perspective of patients with clinically diagnosed TTP-HUS. Semin Hematol. 2004;41(1):60-7.

11. Remuzzi G, Galbusera M, Noris M, Canciani MT, Daina E, Bresin $\mathrm{E}$, et al. von Willebrand factor cleaving protease (ADAMTS13) is deficient in recurrent and familial thrombotic thrombocytopenic purpura and hemolytic uremic syndrome. Blood 2002;100(3):778-85.

12. Furlan M, Robles R, Galbusera M, Remuzzi G, Kyrle PA, Brenner $\mathrm{B}$, et al. von Willebrand factor-cleaving protease in thrombotic thrombocytopenic purpura and the hemolytic-uremic syndrome. N Engl J Med.1998;339(22):1578-84.

13. Choi HS, Cheong HI, Kim NK, Oh D, Park HW. ADAMTS13 gene mutations in children with hemolytic uremic syndrome. Yonsei Med J. 2011;52(3):530-4.

14. Lammle B, Kremer Hovinga JA, Alberio L. Thrombotic thrombocytopenic purpura. J Thromb Haemost. 2005;3(8):1663-75.

15. Tsai HM, Lian EC. Antibodies to von Willebrand factor-cleaving protease in acute thrombotic thrombocytopenic purpura. NEngl J Med.1998;339(22):1585-94.

16. Furlan M, Robles R, Solenthaler M, Wassmer M, Sandoz P, Lammle B. Deficient activity of von Willebrand factor-cleaving protease in chronic relapsing thrombotic thrombocytopenic purpura Blood.1997;89(9):3097-103.

17. Mannucci PM, Peyvandi F. TTP and ADAMTS13: When Is Testing Appropriate? Hematology Am Soc Hematol Educ Program. 2007:121-6.

18. Vesely SK, George JN, Lammle B, Studt JD, Alberio L, El-Harake MA et al. ADAMTS13 activity in thrombotic thrombocytopenic purpura-hemolytic uremic syndrome: relation to presenting features and clinical outcomes in a prospective cohort of 142 patients. Blood. 2003;102(1):60-8.

19. Ardalan M. Rare presentations of cytomegalovirus infection in renal allograft recipients. Nephrourol Mon. 2012;4(2):431-6.

20. Cserti CM, Landaw S, Uhl L. Do infections provoke exacerbations and relapses of thrombotic thrombocytopenic purpura? J Clin Apher. 2007;22(1):21-5.

21. Ardalan MR, Shoja MM, Tubbs RS, Esmaili H, Keyvani H. Postrenal transplant hemophagocytic lymphohistiocytosis and thrombotic microangiopathy associated with parvovirus b19 infection. Am JTransplant. 2008;8(6):1340-4.

22. Ardalan MR, Ansarin K, Hejazi E, Nazemieh M, Safa J. Aspergillosis after renal transplantation. Saudi J Kidney Dis Transpl. 2005;16(3):330-3.

23. Ardalan MR, Tubbs RS, Chinikar S, Shoja MM. Crimean-Congo haemorrhagic fever presenting as thrombotic microangiopathy and acute renal failure. Nephrol Dial Transplant. 2006;21(8):23047.

24. Mannucci PM, Vanoli M, Forza I, Canciani MT, Scorza R. Von Willebrand factor cleaving protease (ADAMTS-13) in 123 patients with connective tissue diseases (systemic lupus erythematosus and systemic sclerosis). Haematologica. 2003;88(8):914-8.

25. Yang EM, Han DK, Baek HJ, Shin MG, Kim YO, Hoon K, et al. TTP with decreased level of ADAMTS-13 activity and increased level of ADAMTS-13 inhibitor in an adolescent. Korean J Pediatr. 2010;53(3).

26. George JN. The association of pregnancy with thrombotic thrombocytopenic purpura-hemolytic uremic syndrome. Curr Opin Hematol. 2003;10(5):339-44.

27. Molvarec A, Rigo JJ, Boze T, Derzsy Z, Cervenak L, Mako V, et al. Increased plasma von Willebrand factor antigen levels but normal von Willebrand factor cleaving protease (ADAMTS13) activity in preeclampsia. Thromb Haemost. 2009;101(2):305-11.

28. van den Born BJ, van der Hoeven NV, Groot E, Lenting PJ, Meijers JC, Levi M, et al. Association between thrombotic microangiopathy and reduced ADAMTS13 activity in malignant hypertension. Hypertension. 2008;51(4):862-6.

29. Noris M, Remuzzi G. Cardiovascular complications in atypical haemolytic uraemic syndrome. Nat Rev Nephrol. 2014;10(3):174-80 